\title{
INVESTIGATIONS ON THE OPTIMUM COMBINATION OF AZIMUTH PHASE CODING AND UP- AND DOWN-CHIRP MODULATION FOR RANGE AMBIGUITY SUPPRESSION
}

\author{
Ryo Natsuaki*, Nida Sakar, Nestor Yague-Martinez, Muriel Pinheiro, Pau Prats-Iraola \\ Microwaves and Radar Institute, German Aerospace Center (DLR), Germany
}

\begin{abstract}
Future spaceborne SAR satellites will achieve both high resolution and wide swath with their multiple receivers. In order to suppress range ambiguities, cyclic up and down chirp and azimuth phase coding have been widely applied. A multiple receiver system has lower pulse repetition frequency to increase the swath width and thus, the effect of azimuth phase coding to mitigate range ambiguities is decreased. In this paper, we reveal the possible combination of the two methods to overcome this drawback.
\end{abstract}

Index Terms - SAR, ambiguity, signal processing, focusing

\section{INTRODUCTION}

An option to achieve both high azimuth resolution and wide swath (HRWS) in Synthetic Aperture Radar (SAR) system is to use a multiple receiver system [1]. The aim of this system is to achieve higher azimuth resolution without increasing the pulse repetition frequency (PRF). This is because the higher PRF results in a narrower observation swath width. Such a solution requires a reconstruction process to achieve single SAR raw data from multiple receivers. However, the reconstruction process leads to additional azimuth ambiguities, whose power depend on the accuracy of the azimuth reconstruction process. On the other hand, recent SAR systems, like the ALOS-2 sensor [2], apply Up-and-Down-Chirp (UDC) method and/or Azimuth Phase Coding (APC) methods to mitigate range ambiguities $[3,4]$. These methods modify the chirp signal so that the desired chirp and interference chirp from outside of the observation area become different. The received chirps must be treated prior to the reconstruction and focusing processes.

The modified chirps must be enough isolated from each other. If the transmit / receive and reconstruction systems are developed improperly, the focused SAR image contains stronger ambiguities in single look complex (SLC) image. For example, APC works less effectively when PRF is low because the variance of Doppler frequency becomes small. We reveal in this paper that improper combination and treatment

${ }^{*} \mathrm{R}$. Natsuaki is a guest scientist by receiving a grant of Overseas Research Fellowships from Japan Society for the Promotion of Science (JSPS), Japan.

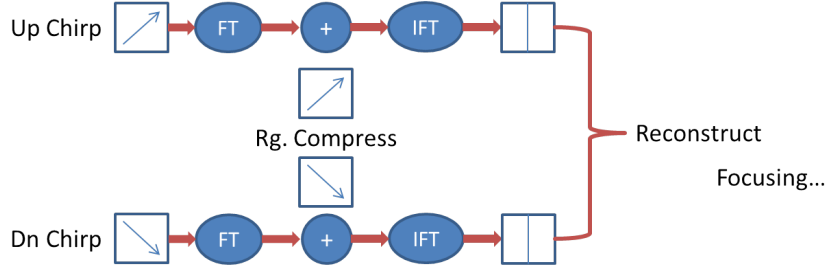

Fig. 1. Schematic diagram of the processing chain.

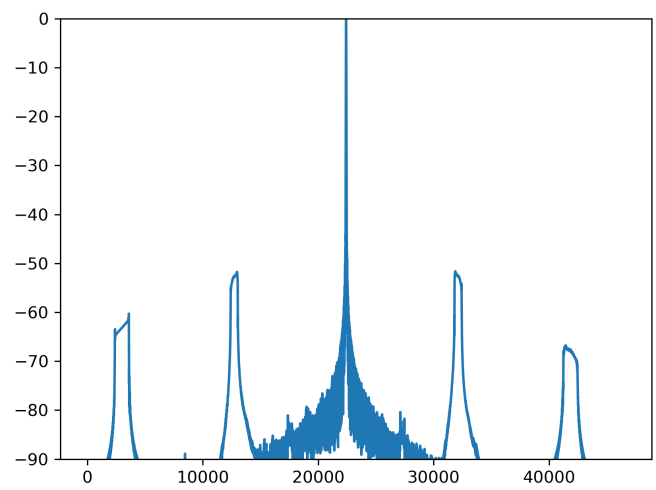

Fig. 2. Power plot in azimuth direction for the point target in main lobe with ALOS-2-like double receiver system.

of UDC and APC raises an additional range and azimuth ambiguity in the reconstruction and focusing processes. On the other hand, if we could apply an optimum chirp pattern, the power of ambiguity becomes lower.

\section{CHIRP MODULATION AND MULTIPLE RECEIVER SYSTEM}

Here, we briefly review the UDC and APC system as well as multiple receiver system. In both UDC and APC methods, SAR transmits orthogonally modified chirps alternatively so that the ambiguous signals will have different chirp pattern from the desired one. The received signals are firstly range 
Table 1. ALOS-2 like SAR parameters for simulation.

\begin{tabular}{c|c}
\hline Observation PRF & $1377.39 \mathrm{~Hz}$ \\
Reconstructed PRF & $2754.77 \mathrm{~Hz}$ \\
Azimuth bandwidth & $2395.48 \mathrm{~Hz}$ \\
Slant range & $1000 \mathrm{~km}$ \\
Platform altitude & $628 \mathrm{~km}$ \\
Platform velocity & $7.6265 \mathrm{~km} / \mathrm{s}$ \\
Pulse length & $72.2195 \mu \mathrm{s}$ \\
Range sampling rate & $104.79 \mathrm{MHz}$ \\
Bandwidth & $79.4 \mathrm{MHz}$ \\
Receiver length in azimuth & $5 \mathrm{~m}$ \\
Distance of phase center & $5 \mathrm{~m}$ \\
\hline
\end{tabular}

Table 2. Lookup table for range compression results in multiple chirp modulation. Note: $\mathrm{D}^{*} / \mathrm{U}^{*}$ and $-\frac{\pi}{2}$ represent range compression and phase shift for APC, respectively.

\begin{tabular}{|c|c||c|c|c|c|}
\hline \multicolumn{1}{|l||}{} & \multicolumn{4}{c|}{ Received Chirp } \\
\hline \multicolumn{2}{|c||}{ Desired } & $\mathrm{D}$ & $\mathrm{U}$ & $\mathrm{D}_{-\frac{\pi}{2}}$ & $\mathrm{U}_{-\frac{\pi}{2}}$ \\
\hline \hline $\mathrm{D}$ & $\mathrm{D}^{*}$ & $\mathrm{DD}^{*}$ & $\mathrm{UD}^{*}$ & $\mathrm{D}_{-\frac{\pi}{2}} \mathrm{D}^{*}$ & $\mathrm{U}_{-\frac{\pi}{2}} \mathrm{D}^{*}$ \\
\hline $\mathrm{U}$ & $\mathrm{U}^{*}$ & $\mathrm{DU}^{*}$ & $\mathrm{UU}^{*}$ & $\mathrm{D}_{-\frac{\pi}{2}} \mathrm{U}^{*}$ & $\mathrm{U}_{-\frac{\pi}{2}} \mathrm{U}^{*}$ \\
\hline $\mathrm{D}_{-\frac{\pi}{2}}$ & $\mathrm{D}_{-\frac{\pi}{2}}^{*}$ & $\mathrm{DD}_{-\frac{\pi}{2}}^{*}$ & $\mathrm{UD}_{-\frac{\pi}{2}}^{*}$ & $\mathrm{D}_{-\frac{\pi}{2}} \mathrm{D}_{-\frac{\pi}{2}}^{*}$ & $\mathrm{U}_{-\frac{\pi}{2}} \mathrm{D}_{-\frac{\pi}{2}}^{*}$ \\
\hline $\mathrm{U}_{-\frac{\pi}{2}}$ & $\mathrm{U}_{-\frac{\pi}{2}}^{*}$ & $\mathrm{DU}_{-\frac{\pi}{2}}^{*}$ & $\mathrm{UU}_{-\frac{\pi}{2}}^{*}$ & $\mathrm{D}_{-\frac{\pi}{2}} \mathrm{U}_{-\frac{\pi}{2}}^{*}$ & $\mathrm{U}_{-\frac{\pi}{2}} \mathrm{U}_{-\frac{\pi}{2}}^{*}$ \\
\hline
\end{tabular}

focused and reconstructed as shown in Fig. 1. The reconstructed raw data is focused afterward.

\subsection{UDC method}

The basic idea of UDC method is to transmit up- and downchirp signal alternatively [3].

$$
\begin{aligned}
U(t) & =\operatorname{Rect}\left[\frac{t}{T_{p}}\right] \exp \left(j \pi K_{r} t^{2}\right) \\
D(t) & =\operatorname{Rect}\left[\frac{t}{T_{p}}\right] \exp \left(-j \pi K_{r} t^{2}\right)
\end{aligned}
$$

where $t$ and $T_{p}$ are time and chirp pulse length, Rect $[\bullet]$ is rectangular function, and $K_{r}$ is chirp rate. The matched filter is applied and range focused in the frequency domain prior to the reconstruction process. That is, in Fig. 1, $D(f)$ and $U(f)$ are range focused with their chirp replica $D^{*}(f)$ and $U^{*}(f)$. When an opposite chirp is received as a range ambiguity, the compressed chirp signal will have doubled chirp duration with a halved intensity and thus, totally de-focused in the SLC image. Note that the up- and down-chirp as an ambiguous signal are supposed to be different in this paper; $D(f) U^{*}(f) \neq U(f) D^{*}(f)$.

\subsection{APC method}

The principle idea of APC is to shift the Doppler spectra of range ambiguity so that it is mitigated during the SAR focusing operation [4]. In order to shift the Doppler spectra for $f_{\mathrm{PRF}} / M$, the $\mathrm{n}(=[0,1, \ldots M])$-th chirp will be modulated by the additional phase $\phi(n)$ such as

$$
\phi(n)=-\frac{\pi}{M} n^{2}
$$

resulting in the range ambiguity signal for $i(\neq n)$ th chirp contains additional Doppler shift. In practice, the value used for $\mathrm{M}$ is 2, which shifts the Doppler spectrum of the odd range ambiguities to $\mathrm{PRF} / 2$, i.e.,

$$
D_{-\frac{\pi}{2}}(t)=D(t) * \exp \left(-j \frac{\pi}{2}\right)
$$

\subsection{Multiple receiver system}

In order to increase azimuth resolution without increasing the PRF, which results in a narrower swath width, some recent researches aim to increase the number of receivers and to reconstruct an identical image [1]. The receivers can be placed either at the displaced part of one satellite or on multiple satellites. Mathematically, raw images of $n$ receivers can be reconstructed as one $n \mathrm{PRF}$ raw data. On the other hand, the reconstruction makes additional azimuth ambiguities in the focused images as shown in Fig. 2.

The figure shows a normalized focusing result for the point target in the main lobe with ALOS-2-like double receiver system. The other parameters are shown in Table 1 . The highest peak is the focused point target while the two peaks at the both end with $-60.3 \mathrm{~dB}$ are the azimuth ambiguities while the other two in the middle with $-51.8 \mathrm{~dB}$ are derived from the reconstruction process.

\section{COMBINATION OF UDC AND APC}

Both UDC and $M=2$ APC systems have two chirp variations. Therefore, the odd (first, third...) near and far range ambiguities can be suppressed but, the even (second, forth...) ones are not. If we could combine both, the number of pattern increases to four. Table 2 is a lookup table for the chirp conversion. The first column is the desired chirp signal, which must be scattered from the main lobe. The second column is the function to convert the desired chirp to down-chirp. The third to sixth columns are the result of conversion for the ambiguous signals.

Theoretically, both UDC and APC expect to be transmitted alternatively. That is, only $\mathrm{D} \rightarrow \mathrm{U}_{-\frac{\pi}{2}}$ is possible as long as we follow the theory strictly. However, this cycle use only two out of four patterns. Therefore, an equivalent cycle is needed. There are only three possible patterns which use all four signals as shown in Table 3. That is, if we start the cycle from $D$, the possible patterns are $3 ! / 2=3$ because the 
Table 3. Lookup tables for chirp cycles and compression results for far range ambiguous chirps.

\begin{tabular}{|c|c|c|c|c|c|}
\hline \multicolumn{6}{|c|}{ UDC only } \\
\hline & $\mathrm{D}$ & $\mathrm{U}$ & $\mathrm{D}$ & $\mathrm{U}$ & $\ldots$ \\
\hline 1 st & $\mathrm{UD}^{*}$ & $\mathrm{DU}^{*}$ & $\mathrm{UD}^{*}$ & $\mathrm{DU}^{*}$ & $\ldots$ \\
\hline \multicolumn{6}{|c|}{ APC only } \\
\hline & $\mathrm{D}$ & $\mathrm{D}_{-\frac{\pi}{2}}$ & $\mathrm{D}$ & $\mathrm{D}_{-\frac{\pi}{2}}$ & $\ldots$ \\
\hline 1 st & $\mathrm{D}_{-\frac{\pi}{2}} \mathrm{D}^{*}$ & $\mathrm{DD}_{-\frac{\pi}{2}}^{*}$ & $\mathrm{D}_{-\frac{\pi}{2}} \mathrm{D}^{*}$ & $\mathrm{DD}_{-\frac{\pi}{2}}^{*}$ & $\ldots$ \\
\hline \multicolumn{6}{|c|}{ Down + phase coded Up only } \\
\hline & $\mathrm{D}$ & $\mathrm{U}_{-\frac{\pi}{2}}$ & $\mathrm{D}$ & $\mathrm{U}_{-\frac{\pi}{2}}$ & $\ldots$ \\
\hline 1 st & $\overline{\mathrm{U}_{-\frac{\pi}{2}} \mathrm{D}^{*}}$ & $\overline{\mathrm{DU}_{-\frac{\pi}{2}}^{*}}$ & $\overline{\mathrm{U}_{-\frac{\pi}{2}} \mathrm{D}^{*}}$ & 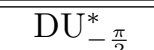 & ב... \\
\hline \multicolumn{6}{|c|}{$\mathrm{UDC}+\mathrm{APC}$ cycle 1} \\
\hline & $\mathrm{D}$ & $\mathrm{D}_{-\frac{\pi}{2}}$ & $\mathrm{U}$ & $\mathrm{U}_{-\frac{\pi}{2}}$ & $\ldots$ \\
\hline $1 \mathrm{st}$ & $\mathrm{U}_{-\frac{\pi}{2}} \mathrm{D}^{*}$ & $\mathrm{DD}_{-\frac{\pi}{2}}^{*}$ & $\mathrm{D}_{-\frac{\pi}{2}} \mathrm{U}^{*}$ & $\mathrm{UU}_{-\frac{\pi}{2}}^{*}$ & $\ldots$ \\
\hline 2 nd & $\mathrm{UD}^{*}$ & $\mathrm{U}_{-\frac{\pi}{2}} \mathrm{D}_{-\frac{\pi}{2}}^{*}$ & $\mathrm{DU}^{*}$ & $\mathrm{D}_{-\frac{\pi}{2}} \mathrm{U}_{-\frac{\pi}{2}}^{*}$ & $\ldots$ \\
\hline $3 \mathrm{rd}$ & $\mathrm{D}_{-\frac{\pi}{2}} \mathrm{D}^{*}$ & $\mathrm{UD}_{-\frac{\pi}{2}}^{*}$ & $\mathrm{U}_{-\frac{\pi}{2}} \mathrm{U}^{*}$ & $\mathrm{DU}_{-\frac{\pi}{2}}^{*}$ & $\ldots$ \\
\hline \multicolumn{6}{|c|}{$\mathrm{UDC}+\mathrm{APC}$ cycle 2} \\
\hline & $\mathrm{D}$ & $\mathrm{U}$ & $\mathrm{U}_{-\frac{\pi}{2}}$ & $\mathrm{D}_{-\frac{\pi}{2}}$ & $\ldots$ \\
\hline 1 st & $\mathrm{D}_{-\frac{\pi}{2}} \mathrm{D}^{*}$ & 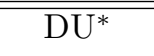 & 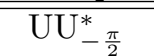 & $\mathrm{U}_{-\frac{\pi}{2}} \mathrm{D}_{-\frac{\pi}{2}}^{*}$ & $\ldots$ \\
\hline 2 2nd & $\mathrm{U}_{-\frac{\pi}{2}} \mathrm{D}^{*}$ & $\mathrm{D}_{-\frac{\pi}{2}} \mathrm{U}^{*}$ & $\mathrm{DU}_{-\frac{\pi}{2}}^{*}$ & $\mathrm{UD}_{-\frac{\pi}{2}}^{*}$ & $\ldots$ \\
\hline $3 \mathrm{rd}$ & $\mathrm{UD}^{*}$ & $\mathrm{U}_{-\frac{\pi}{2}} \mathrm{U}^{*}$ & $\mathrm{D}_{-\frac{\pi}{2}} \mathrm{U}_{-\frac{\pi}{2}}^{*}$ & $\mathrm{DD}_{-\frac{\pi}{2}}^{*}$ & $\ldots$ \\
\hline \multicolumn{6}{|c|}{ UDC + APC cycle 3} \\
\hline & $\mathrm{D}$ & $\mathrm{U}$ & $\mathrm{D}_{-\frac{\pi}{2}}$ & $\mathrm{U}_{-\frac{\pi}{2}}$ & $\ldots$ \\
\hline $1 \mathrm{st}$ & $\mathrm{U}_{-\frac{\pi}{2}} \mathrm{D}^{*}$ & $\overline{\mathrm{DU}^{*}}$ & $\mathrm{UD}_{-\frac{\pi}{2}}^{*}$ & $\mathrm{D}_{-\frac{\pi}{2}} \mathrm{U}_{-\frac{\pi}{2}}^{*}$ & 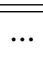 \\
\hline 2nd & $\mathrm{D}_{-\frac{\pi}{2}} \mathrm{D}^{*}$ & $\mathrm{U}_{-\frac{\pi}{2}} \mathrm{U}^{*}$ & $\mathrm{DD}_{-\frac{\pi}{2}}^{*}$ & $\mathrm{UU}_{-\frac{\pi}{2}}^{*}$ & $\ldots$ \\
\hline 3rd & $\mathrm{UD}^{*}$ & $\mathrm{D}_{-\frac{\pi}{2}} \mathrm{U}^{*}$ & $\mathrm{U}_{-\frac{\pi}{2}} \mathrm{D}_{-\frac{\pi}{2}}^{*}$ & $\mathrm{DU}_{-\frac{\pi}{2}}^{*}$ & $\ldots$ \\
\hline
\end{tabular}

opposite cycle is equal to each other. Table 3 provides the conversion results for the ambiguious signals. As discussed in Sec. 2, APC methods do not work effectively when PRF is low, and in $n$-receiver system, the actual PRF is $1 / n$ of the processing PRF. Therefore, at least the first ambiguous signals must be mitigated with UDC method. In short, cycle 1 and 2 are less effective than cycle 3 in a qualitative manner, because some of their ambiguous chirps are mitigated only with APC.

\section{EXPERIMENTAL RESULTS}

In this section, we compared the effectiveness of the possible 3 cycles discussed in the previous section. We analyzed the point target analysis placed in the first far-range ambiguity. We calculated the relative power from a point target in the main lobe (Fig. 2) in order to evaluate the power of the ambiguous chirps. Note that the effect of antenna elevation pattern is ignored in this experiment so that we can investigate the effect of the chirp modulation.
Figure 3 shows the power plot of conventional two chirp pattern in azimuth direction. The peak power of UDC, APC and $\mathrm{D} \rightarrow \mathrm{U}_{-\frac{\pi}{2}}$ cycle are $-50.8 \mathrm{~dB},-33.1 \mathrm{~dB}$ and $-50.8 \mathrm{~dB}$ respectively. APC was less effective than UDC because of the low PRF. There was no big difference between UDC and $\mathrm{D} \rightarrow \mathrm{U}_{-\frac{\pi}{2}}$ cycle. The peak power of UDC is slightly stronger than the peak of azimuth ambiguity $(-51.8 \mathrm{~dB})$.

Figure 4 shows the results for the three combination cycles. Their peak powers were (a) $-19.8 \mathrm{~dB}$, (b) $-30.7 \mathrm{~dB}$ and (c) $-54.9 \mathrm{~dB}$. That is, cycle 3 has $4.1 \mathrm{~dB}$ lower peak than the UDC case while other cycles were worse than UDC. In summary, combination of four chirp patterns decreases the peak of range ambiguity in this condition. In addition, it can reduce the power of second range ambiguity, which is impossible for the conventional cycles.

\section{CONCLUSION}

In this paper, we revealed the existence of the optimum combination for range ambiguity suppression using up-and-downchirp and azimuth phase coding. Simulation results show that the worst combination shows worse results than applying only one of them. At the same time, an expected drawback of APC, the amount of the Doppler shift depends on the actual PRF, was clearly seen. On the other hand, range ambiguities will be suppressed weaker than the azimuth ambiguity of the mainlobe in the optimum case. In the best case, $\mathrm{D} \rightarrow \mathrm{U} \rightarrow \mathrm{D}_{-\frac{\pi}{2}} \rightarrow \mathrm{U}_{-\frac{\pi}{2}}$ pattern, the first to third range ambiguities can be mitigated more than conventional ones.

\section{REFERENCES}

[1] G. Krieger, N. Gebert, and A. Moreira, "Unambiguous SAR signal reconstruction from nonuniform displaced phase center sampling," IEEE Geoscience and Remote Sensing Letters, vol. 1, no. 4, pp. 260-264, oct 2004.

[2] Yukihiro Kankaku, Masakazu Sagisaka, and Shinichi Suzuki, "PALSAR-2 launch and early orbit status," in International Geoscience and Remote Sensing Symposium (IGARSS) 2014, 2014, pp. 3410-3412.

[3] J. Mittermayer and J.M. Martinez, "Analysis of range ambiguity suppression in SAR by up and down chirp modulation for point and distributed targets," in 2003 IEEE International Geoscience and Remote Sensing Symposium (IGARSS), 2003, pp. 4077-4079.

[4] Jorgen Dall and Anders Kusk, "Azimuth phase coding for range ambiguity suppression in SAR," in 2004 IEEE International Geoscience and Remote Sensing Symposium (IGARSS), 2004, pp. 1734-1737. 


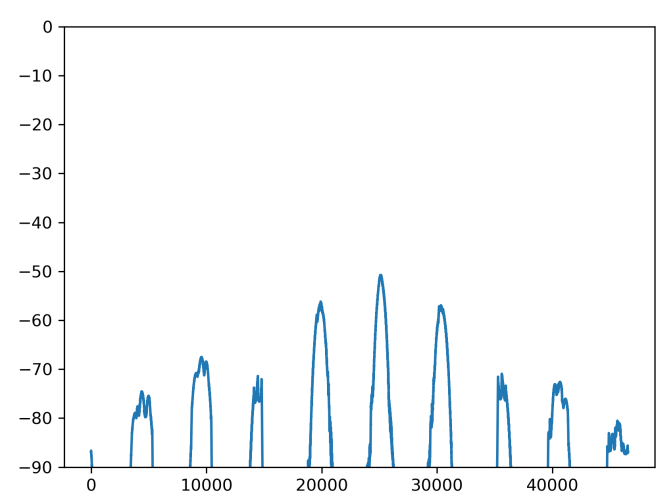

(a)

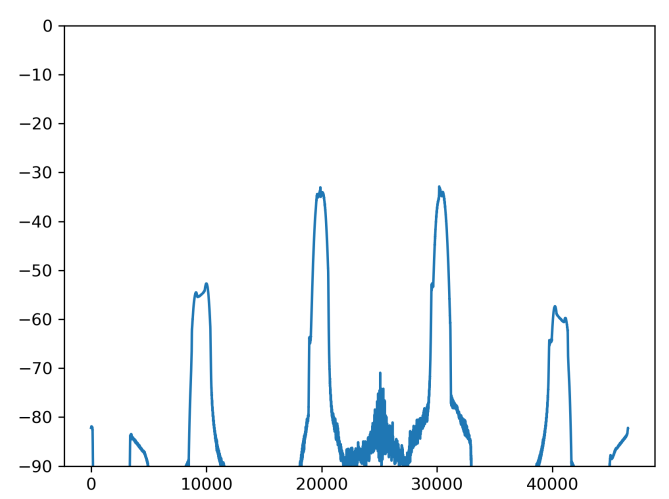

(b)

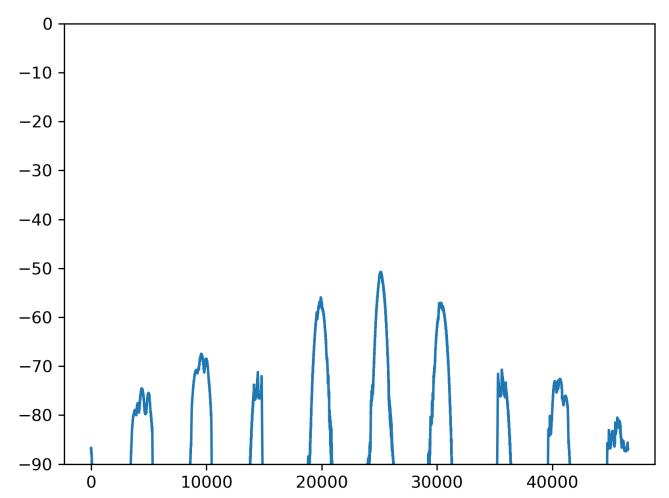

(c)

Fig. 3. Comparison of range ambiguity appearance. (a) UDC only, (b) APC only and (c) Down + phase coded Up $\left(\mathrm{D} \rightarrow \mathrm{U}_{-\frac{\pi}{2}}\right)$ only.

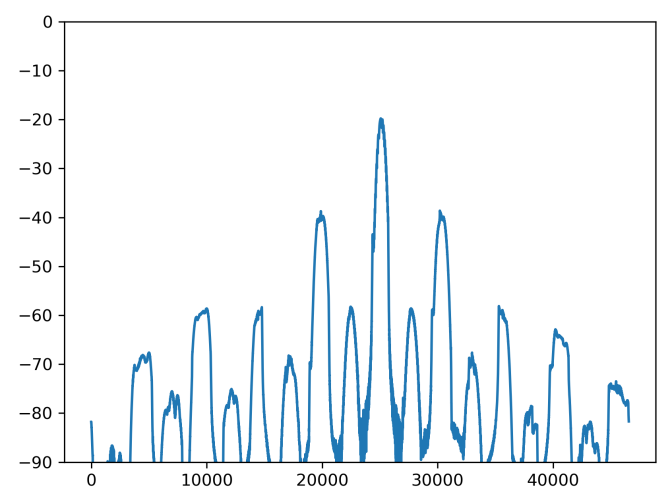

(a)

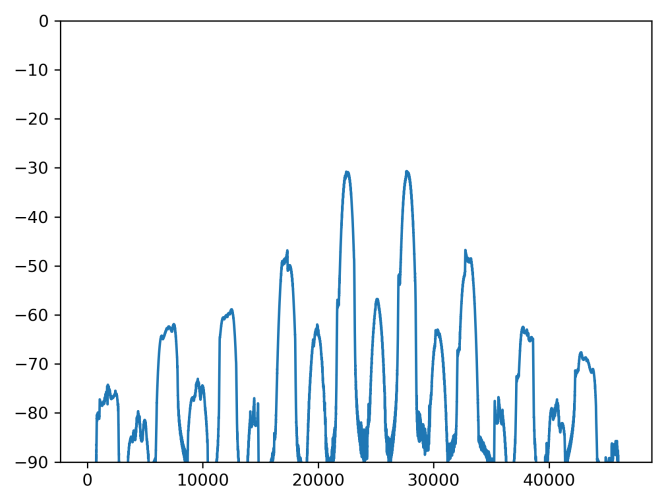

(b)

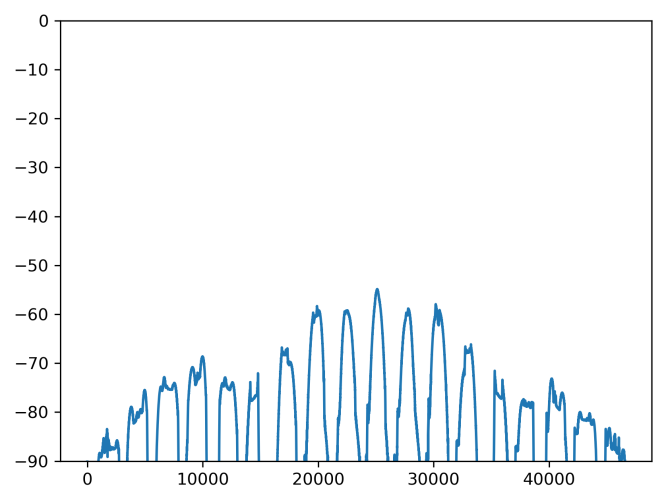

(c)

Fig. 4. Comparison of range ambiguity appearance. (a) cycle $1 \quad\left(\mathrm{D} \rightarrow \mathrm{D}_{-\frac{\pi}{2}} \rightarrow \mathrm{U} \rightarrow \mathrm{U}_{-\frac{\pi}{2}}\right)$, (b) cycle $2 \quad\left(\mathrm{D} \rightarrow \mathrm{U} \rightarrow \mathrm{U}_{-\frac{\pi}{2}} \rightarrow \mathrm{D}_{-\frac{\pi}{2}}\right)$ and (c) cycle 3 $\left(\mathrm{D} \rightarrow \mathrm{U} \rightarrow \mathrm{D}_{-\frac{\pi}{2}} \rightarrow \mathrm{U}_{-\frac{\pi}{2}}\right)$. 